\title{
Programa familias fuertes: amor y límites para la prevención del consumo de drogas y alcohol
}

\author{
Strong family's program: love and limits for the prevention of drug and alcohol \\ consumption
}

\author{
Programa Famílias Fortes: amor e limites para a prevenção do consumo \\ de drogas e álcool
}

\author{
Johanna Alemán Ríos \\ yoy77895599@hotmail.com \\ https://orcid.org/0000-0002-7239-5561
}

Universidad Autónoma "Juan Misael Saracho", Tarija, Bolivia

RESUMEN

En la prevención de conductas de riesgos, los padres cumplen la función de ayudar a sus hijos a afrontar la vida adulta, para poder integrarse a la sociedad. El estudio tuvo como objetivo implementar el programa "familias fuertes: amor y límites", para prevenir el consumo de alcohol y otras drogas, dirigido a padres de familia de Unidades Educativas en la ciudad de Tarija. La metodología que se usó fue método activo participativo. Las técnicas utilizadas fueron: audiovisuales, dinámica de integración, de grupo, de análisis y reflexión. Mientras que los instrumentos fueron: manual del programa familias fuertes, manual de recursos para el facilitador, encuestas para padres. Se obtuvo como resultado, que los padres con el programa desarrollaron habilidades para relacionarse de mejor manera con sus hijos. Por lo que se concluye, que el programa permite orientar a los padres para educar a sus hijos referentes al alcohol y drogas.

Palabras clave:

Prevención de drogas y alcohol; crianza autoritaria; crianza permisiva; crianza efectiva
ABSTRACT

RESUMO

In the prevention of risky behaviors, parents fulfill the function of helping their children to face adult life, in order to be able to integrate into society. The objective of the study was to implement the program "strong families: love and limits", to prevent the consumption of alcohol and other drugs, aimed at parents of Educational Units in the city of Tarija. The methodology used was the active participatory method. The techniques used were: audiovisual, integration, group, analysis and reflection dynamics. While the instruments were: strong families program manual, resource manual for the facilitator, parent surveys. It was obtained as a result, that the parents with the program developed skills to relate in a better way with their children. Therefore, it is concluded that the program allows orienting parents to educate their children about alcohol and drugs.

Key words:

Drug and alcohol prevention, authoritarian parenting; permissive parenting; effective parenting
Na prevenção de comportamentos de risco, os pais cumprem a função de ajudar os filhos a enfrentar a vida adulta, de forma a poderem do estudo foi implementar o programa "Famílias fortes: amor e limites", de prevenção ao consumo de álcool e outras drogas, dirigido a pais de Unidades de Ensino da cidade de Tarija. A metodologia utilizada foi o método participativo ativo. As técnicas utilizadas foram: audiovisual, integração, dinâmica de grupo, análise e reflexão. Enquanto os instrumentos foram: manual do programa para famílias fortes, manual de recursos para - facilitador, pesquisas com os pais. Obteve-se como resultado, que os pais com o programa desenvolveram habilidades para se relacionar melhor com seus filhos. Portanto, conclui-se que o programa permite orientar os pais a educar seus filhos sobre álcool e drogas.

Palavras-chave:

Prevenção de drogas e álcool; parentalidade autoritária; paternidade permissiva; paternidade eficaz se inserir na sociedade. $\mathrm{O}$ objetivo 


\section{INTRODUCCIÓN}

El desarrollo de una vida humana es posible gracias a diversos factores naturales como sociales; siendo el ser humano, un individuo social, por ende, necesita de otros para crecer. A causa de ello la persona al nacer se encuentra dentro de un grupo social que es básico y fundamental para su desarrollo en sociedad, este grupo está constituido por la "familia".

Para Kumpfer (1999), la familia juega un papel preponderante a la hora de prever conductas de riesgo en los jóvenes, ya que como lo indica es:

Un ambiente familiar positivo, afirmado en los vínculos familiares, con una supervisión adecuada de madres y padres en el comportamiento de los hijos, una comunicación de los valores familiares pro-sociales, son aspectos que protegen a los jóvenes de desarrollar conductas de riesgo, como el consumo de sustancias ilícitas, la delincuencia y el sexo precoz o sin protección (p. 52).

Dentro de la prevención de conductas de riesgos, los padres cumplen la función de ayudar a sus hijos a afrontar la vida adulta, preparándolos para ella y así poder integrarse de la mejor manera a la sociedad. Además de brindarles una estabilidad social, emocional y económica, ayudan a que sus hijos aprendan las reglas básicas de la sociedad en la que viven, les enseñan a socializar, a escuchar, comunicar, conocer y desarrollar los derechos y deberes determinantes de esa sociedad.

Cuando no se crean estos vínculos de afecto, protección, acompañamiento, comprensión, comunicación y enseñanza, hay mayor probabilidad que los hijos puedan tener conductas erróneas, como el consumo de alcohol y otras drogas, delincuencia, sexo precoz o sin protección, y un sin fin de otras conductas de riesgo.
En este sentido, a nivel internacional según el Informe Mundial sobre las Drogas- UNODOC (2016): "se calcula que 1 de cada 20 adultos, es decir, alrededor de 250 millones de personas de entre 15 y 64 años, consumieron algún tipo de droga en el 2015" (p.9).

Siendo estos datos alarmantes ya que, entre un tercio y la mitad de los adolescentes consume alcohol, sin tener en cuenta las consecuencias que esto les puede acarrear, como ser: la muerte y lesiones no intencionales asociadas con llevar a cabo otras labores de riesgo luego de beber, por ejemplo: homicidio, violencia, intentos de suicidio, abuso sexual, comportamiento sexual de riesgo, vandalismo, daños a la propiedad, entre otros.

La Organización Mundial de Salud (2013) expone que, a nivel nacional en Bolivia, el consumo de alcohol ubica al país en el medio de la región promedio con 5,9 litros pér cápita. Siendo los jóvenes entre 15 y 17 años los que mayormente consumen. Según este informe, entre las causas para el incremento están: la libre oferta de alcohol y la falta del fortalecimiento de los valores, además que en la sociedad es común asociar este consumo a diferentes fiestas patronales, fechas cívicas y otras festividades.

Bolivia es un país de profunda tradición, respecto al consumo de alcohol, este fenómeno que se halla incorporado a sus ritos y tradiciones, el consumo de bebidas alcohólicas en su conjunto mantiene una tendencia creciente, sobre todo en los jóvenes cuyo inicio es a edades muy tempranas, generando una importante alarma social; estos adolescentes y jóvenes perciben al alcohol como un facilitador de las relaciones sociales, un instrumento de cohesión entre el grupo de iguales y un elemento básico para la diversión, es decir un hecho simplemente cultural, minimizando las condicionantes y consecuencias de su ingesta, ignorando completamente los factores que intervienen en esta problemática multicausal. 
A nivel local, Tarija no se queda atrás; en la actualidad, ocupa el primer lugar en el consumo del alcohol, según las estadísticas que maneja el Instituto dePrevención, Tratamiento, Rehabilitación e Investigación de Drogodependencias y Salud Mental (INTRAID).

De esta forma, la investigación tiene por objetivo principal, implementar el Programa "Familias Fuertes: Amor y Límites", con la finalidad de prevenir el consumo de alcohol y otras drogas, dirigido a padres de familia de Unidades Educativas en la ciudad de Tarija, durante la gestión 2017.

Con el programa "Familias Fuertes: Amor y Límites", se busca la intervención primaria, para capacitar a padres de familia en habilidades de crianza y relaciones familiares, para prevenir conductas de riesgo, en adolescentes de 10 a 14 años de edad.

\section{Marco Teórico}

\section{Programas familias fuertes}

\section{Modelo ecológico}

Este modelo fue desarrollado por Bronfenbrenner (1982) para entender el desarrollo humano sistémicamente, cuya centralidad recae en la persona (p. 22).

Sefundamenta en la relación dinámicay circular que existe entre la persona y su medio ambiente familiar, comunitario y social. Esta relación es transformadora. La persona es impactada por el medio adoptando conductas estereotipadas y modeladas. Y la persona a su vez, puede contribuir a modificar el medio, contribuyendo al cambio de patrones, estilos de vida y entornos. Es un proceso dinámico y circular. Este abordaje permite mirar los fenómenos sociales desde una perspectiva de factores determinantes estructurales que influyen nocivamente $y$ son conocidos como predisponentes o distales. Los factores de riesgo que operan más a nivel comunitario se los reconoce como facilitadores o contextuales. Los factores de riesgo que operan a nivel familiar son llamados reforzadores o proximales.

\section{Teorías para el cambio de la conducta}

Usar teorías orientadas al cambio de la conducta para aplicar eficazmente el Programa Familias Fuertes: Amor y Límites, es indispensable para producir el impacto previsto en la aplicación de este programa. Sin el uso de teorías en la aplicación del programa, no es posible alcanzar los resultados para los cuales fue diseñado este. Tampoco se alcanza los resultados previstos si no se respeta la fidelidad del programa, cualquier cambio de añadidos o supresiones en su metodología solo pueden ser realizados por las instituciones que le dieron origen, sobre la base de evidencias recogidas en estudios de impacto.

Brienbauer y Maddaleno (2008) indicaron:

Las teorías y los modelos son útiles para las etapas de planificación, implementación y evaluación de una intervención, pues: a) aportan las herramientas necesarias para identificar qué es lo que deben conocer los planificadores de programas antes de diseñar e implementar un programa de intervención basado en la prevención; b) ofrecen una idea acerca de cómo seleccionar las intervenciones adecuadas para cada programa, de que lleguen a los adolescentes y logren algún impacto en ellos y en su entorno; y c)ayudan a identificar cuáles son los aspectos que se deben monitorear, medir o comparar en la evaluación de un programa (p.18). 


\section{Teoría de la autorregulación}

Muss (1996) señala que la autorregulación es un proceso dinámico orientado a definir y lograr metas de salud o de desarrollo y está determinado por factores biológicos y factores ambientales. La capacidad de autorregulación aumenta con la edad, a medida que el lóbulo pre frontal del cerebro se desarrolla, es una habilidad que se aprende paulatinamente durante la adolescencia (p.58). Debido a las diferencias biológicas y ambientales, algunos adolescentes adquieren la capacidad de autorregularse con mayor facilidad que otros. Esta se asocia con menor uso de substancias y mejor rendimiento.

La autorregulación se concreta cuando una persona es capaz de monitorear su propio repertorio de conductas, utilizando señales y retroalimentaciones del mundo exterior. Esto requiere de capacidades y habilidades para el establecimiento de metas, capacidad de automotivación y autodirección para alcanzar las mismas. Asimismo, "se necesita de una capacidad para la autoevaluación de los errores, las debilidades y los obstáculos" (Bartholomew, 2005).

\section{Teoría de modelos de crianza efectiva}

Los modelos de crianza están basados en conceptos de estilos de demanda y atención. Predice que del cruce entre estilos de alta demanda y alta atención resulta el Modelo de Autoridad que es el modelo más protector y eficaz.

\section{Clasifica en cuatro los estilos de crianza}

Autoridad. Significa alta demanda y alta atención. Es el modelo más eficaz. Se caracteriza por padres involucrados, con capacidad de respuesta inmediata, con un nivel de exigencia, en donde se combina, la calidez, la firmeza y la justicia. Fomenta los límites sin violencia.
Permisivo. Representa una alta atención y poca demanda. Este modelo es menos eficaz, puede ser cálido y exigente, pero no coloca límites a la conducta.

Autoritario. Se basa en una alta demanda y poca atención. Este modelo en la crianza no es eficaz. Provoca la necesidad de transgresión de la conducta. Los hijos no se sienten valorados, ni queridos, ni confiados, pero además se sienten culpables.

No involucrado. Se caracteriza por la poca demanda y la poca atención. Este es un modelo ausente, no responde, no se involucra, no es exigente ni firme. Con este modelo los hijos no se sienten valorados ni queridos.

La mayoría de los investigadores coincide en que "la crianza efectiva se apoya en cuatro componentes principales: la calidez, estructura, apoyo de la autonomía y el apoyo al desarrollo"( Brienbauer y Maddaleno, 2008, p.141)

La calidez. En los padres cálidos en la relación con sus hijos desarrollan un vínculo fuerte, se puede observar que generan hijos más complacientes y más abiertos a escucharlos.

Estructura. Es la medida en que los adolescentes tienen expectativas y normas para su conducta. Los padres que aplican la crianza efectiva: a) son firmes en los límites que han establecido para sus hijos y establecen normas para su conducta; a la vez, crean expectativas coherentes con las necesidades y capacidad de desarrollo del niño, y b) reconocen que existen límites negociables, que enseñan responsabilidad, y límites no negociables, que protegen a los jóvenes.

Apoyo de la autonomía. Se sostiene en el modo en que los padres aceptan y fomentan la individualidad del adolescente. Los padres que se adhieren a la disciplina son más proclives (que los padres autoritarios o sobreprotectores) a dar 
a sus hijos una independencia gradual a medida que crecen, brindándoles oportunidades para que desarrollen su autonomía, pero, al mismo tiempo, proporcionándoles normas y límites claros para su conducta y las pautas respectivas.

Apoyo al desarrollo. Los padres que favorecen las capacidades de desarrollo cognitivo y emocional y de desarrollo de intereses del adolescente tienen mayor probabilidad de mejorar la comunicación y la confianza con sus hijos. Así, la empatía de los padres ayuda a guiar a los hijos adolescentes en el descubrimiento de estrategias más saludables para regular sentimientos intensos de tristeza, miedo, envidia, celos, ira y excitación descontrolada, al tiempo que deben proporcionarles modelos positivos que puedan seguir para desarrollar su propia habilidad de empatía.

\section{Teoría social cognitiva}

Bandura (1983) plantea la interacción de factores determinantes de la conducta. Para esta teoría la conducta representa un modelo dinámico y recíproco impactado por estos factores, coincidiendo con el abordaje ecológico. Parte de tres principios: la conducta recibe la influencia tanto de factores del entorno social como de factores personales. Los factores del entorno social desempeñan un papel significativo en el inicio de las conductas, pero su influencia se produce a través de las cogniciones personales. La conducta del adolescente no solo recibe la influencia de los factores personales y del entorno social, sino que también influye sobre estos.

\section{Teoría del vínculo}

Pichon (2009) lo define como:

Estructura dinámica en continuo movimiento que engloba por igual a la persona con otras personas. Es una relación particular con una persona y esta relación particular resulta una conducta más o menos fija, la cual forma una pauta de conducta que tiende a repetirse automáticamente, tanto en la relación interna como en la relación externa (p. 35).

\section{Esto puede ser:}

Vínculos primarios. Son aquellos lazos primarios que se dan en el marco familiar, sean consanguíneos o no.

Vínculos secundarios. Se construyen fuera del núcleo familiar, de parentesco o de personas significativas. Son lazos secundarios que contribuyen en el proceso de individuación infantil y consolidación de la identidad adolescente. Ayudan en la regulación social. Son un indicador de salud mental.

\section{Contenido temático del programa familias fuertes}

\section{Comunicación}

Según Cangas y Moldes (2009) desde los primeros momentos de la vida, la comunicación constituye una parte primordial en el desarrollo de toda persona, no solamente para satisfacer las demandas básicas de alimento, vestido, seguridad, entre otros, sino también otras más ligadas a la exploración, al placer o a la adaptación. Para ello, el contacto físico que se recibe desde pequeño contribuye a un buen desarrollo físico y emocional.

En cada familia existe una diferente modalidad de comunicación: hay familias con comunicación indirecta, vaga y poco sincera, en las cuales existe mucho conflicto, la autoestima de sus integrantes se ve disminuido, las reglas son rígidas, inhumanas, fijas e inmutables, por lo cual el enlace de la familia con la sociedad es poco accesible.

Por otro lado, hay familias en las que la comunicación es directa, clara, específica y sincera, 
en éstas hay poco conflicto y se da una resolución adecuada cuando la hay, existe mayor interés entre los asuntos familiares, sus integrantes tienen una sana autoestima y las reglas son flexibles, humanas, adecuadas y sujetas a cambio, por lo tanto, su enlace con la sociedad es abierto y confiado y está fundamentado en la elección.

Es importante que los padres se puedan comunicar abierta y efectivamente con sus hijos. Este tipo de comunicación beneficia no solo a los niños, sino también a cada miembro de la familia.

\section{Afecto y cuidado}

El afecto y el cuidado son "procesos de interacción social entre dos o más organismos y pueden ser entendidos como algo que puede darse a otro, algo que fluye y se traslada de una persona a otra" (González, Hidalgo, y Moreno, 1998, p.50).

El afecto es la base del desarrollo de las personas. Todo lo que aprendemos lo recibimos a través de las relaciones que establecemos y de la seguridad que éstas nos ofrecen. Estas relaciones garantizan no solo nuestra supervivencia física, sino la posibilidad de madurar a nivel psicológico, social y cognitivo.

\section{Control parental frente al mal comportamiento}

El control parental es definido como, el conjunto de límites, reglas, restricciones y regulaciones que los padres tienen para sus hijos, y el conocimiento de las actividades que estos realizan (Barber y Oslen, 1997, p.12).

A su vez, Brazalton (2006) señala que, después del amor, el segundo regalo más importante de los padres al niño es el sentido de la disciplina. Disciplina significa enseñar, no castigar.

Las normas le permiten saber a las personas qué está bien y qué está mal, establecen qué sucederá cuando se respetan y qué cuando no.
Sirven para circunscribir las características de los comportamientos y de las relaciones entre las personas. Nos orientan para saber qué esperar y qué no en situaciones diversas. El conjunto de normas de una familia y de un grupo social constituye un marco de confianza que brinda, tanto a los niños como a los adultos, la claridad y la estabilidad necesarias para crecer y relacionarse con los demás.

\section{Información de padres a hijos sobre el consumo de alcohol y drogas}

El consumo de tabaco, alcohol y otras drogas suele iniciarse durante la adolescencia. De hecho, no hay prácticamente ningún adulto consumidor de alguna de estas sustancias que no empezara antes de los 18 años. Por eso es conveniente retrasar al máximo la edad de inicio del uso de tabaco, alcohol y otras drogas. Además, los adolescentes que empiezan muy jóvenes a fumar tabaco y a beber alcohol tienen más probabilidades de probar y consumir otras drogas más adelante. Por lo tanto, si se evita el consumo de tabaco y alcohol mientras se es muy joven, se reduce también el riesgo de llegar a consumir otras drogas.

La existencia de información cierta, oportuna, sin tabúes, que ayuden a destruir mitos falsos, con la transmisión de toda la información necesaria se podrá ayudar a que el adolescente conozca los efectos negativos sobre esta problemática, y tome las mejores decisiones para su vida, por eso es de vital importancia que los padres se informen, solo así podrán ayudar a sus hijos.

\section{Dinámica familiar}

Es importante que cada familia crea un ambiente familiar con recursos imprescindibles para evitar conductas de riesgo; cuando se menciona recursos familiares, se hace referencia, a aquellos aspectos sociales, emocionales y físicos que hacen que la 
familia sea menos sensible al riesgo y más capaz de afrontar satisfactoriamente los factores estresantes, facilitando la adaptación individual y familiar. Varias investigaciones sustentan este factor, como señala Malkus (1994), los jóvenes que proceden de familias con más recursos son menos vulnerables al involucramiento del abuso de drogas.

\section{MÉTODO}

La metodología que se utilizó para el desarrollo de la investigación fue el método activo participativo.

Teniendo en cuenta las características de la investigación y constituyéndose desde una perspectiva socio educativa, el método empleado fue activo participativo, mismo que se utilizó, debido a su flexibilidad, lo cual permitió que los participantes se conviertan en agentes activos en la construcción, reconstrucción del conocimiento y no como agentes pasivos, simplemente receptores, promoviendo y procurando la participación activa y protagónica de todos los integrantes del grupo, incluyendo al facilitador (practicante) en el proceso de enseñanza-aprendizaje; asimismo, nos permitió la reflexión y análisis en cada una de las sesiones desarrolladas, asegurando su participación individual y grupal, al mismo tiempo desarrollar un trabajo flexible, individual y colectivo de análisis a través del intercambio de ideas y experiencias vividas.

Las técnicas que se aplicaron para el desarrollo de la práctica, fueron diseñadas y seleccionadas de tal manera que permitieron fortalecer el proceso del desarrollo del programa "familias fuertes: amor y límites", como ser: técnicas motivadoras, movilizantes, lúdicas, creativas y democráticas, las cuales partieron de los sentimientos y pensamientos; actitudes y vivencias cotidiana de los participantes para generar la posibilidad de transformación personal y cambio cultural, que se buscó a través del mencionado programa.

Las técnicas que se utilizaron fueron las siguientes: audiovisuales, dinámica de animación e integración, dinámica de grupo, dinámica de análisis y reflexión, lluvias de ideas, lúdicas. Mientras que los instrumentos fueron: manual del programa familias fuertes, manual de recursos para el facilitador, encuestas para padres, madres o tutores.

A su vez, los materiales que se utilizaron para la aplicación del programa Familias Fuertes fueron los siguientes: televisor y lector de DVD, videos del programa, rotafolios o papelógrafos, lista de asistencia de los padres, tarjetas de identificación, cinta adhesiva, materiales de escritorio (lápices, marcadores, tijeras, reglas, pegamento, hojas de papel blanco y de colores, etc.), juego de cartas (naipes), recortes de revistas (trabajo, pasatiempos, amigos-familia, salud, etc.), retazos de tela para cubrir los ojos, sobres para cartas. Entre otros.

\section{RESULTADOS}

\section{Descripción sistematizada del programa de prevención}

El programa fue dirigido a los padres y se llevó a cabo en 7 sesiones; sin embargo, para este estudio solo se tomaron 4

\section{Módulo para los padres}

\section{Sesión 1}

Tema 1. Metas y cualidades de los adolescentes.

Se proyectó un video que contó con escenas de padres, hablando entre ellos y otras personas, hablando con sus hijos adolescentes, sobre diferentes actividades que realizaron para su entretenimiento y recreación. 
La actividad que se realizó tuvo como fin indagar sobre las cualidades de desearían que sus hijos tuvieran. Entre las opciones que dieron fueron; "yo quiero que sea respetuoso con los mayores", fueron las palabras de una mamá, "que sea responsable con sus tareas de la escuela, que me ayude en la casa" señaló un papá. Otras cualidades que señalaron fueron: ser solidario, honesto, que ayude a sus hermanos, que sea confiable, solidario, obediente, entre otros.

\section{Tema 2. Resultados de la crianza autoritaria}

Se proyectó un video que contó con escenas de padres, hablando entre ellos y otras personas hablando con sus hijos adolescentes, sobre lo que representa una crianza autoritaria.

La actividad que se realizó tuvo como fin que los padres dieran su punto de vista sobre el significado de la crianza autoritaria, tomando en cuenta el video visto con anterioridad.

Se obtuvo como respuesta de los padres: "es mala... mi hija no me tendrá confianza, me ocultará las cosas, se volverá rebelde...por eso hay que tenerles confianza a los hijos, se les debe escuchar, entenderlos..." y;

Es como si yo fuera el presidente de mi casa, donde todo lo que yo digo es ley, todos deben obedecerme, hacer lo que yo diga...esto es malo porque mis hijos me tendrán miedo, y yo no quiero eso, porque yo quiero a mis hijos, además ya no querrán contarme nada..., sí licenciada a veces yo creo que me porto así, pero lo hago porque creo que también los papás de antes fueron así conmigo. $Y$ también porque ahora los hijos ya no hacen caso como antes.

\section{Tema 3. Resultados de la crianza permisiva}

Se proyectó un video que contó con escenas de padres, hablando entre ellos y otras personas hablando con sus hijos adolescentes, sobre lo que representa una crianza permisiva.

La actividad que se realizó tuvo como fin, que, los padres dieran su punto de vista con respecto a la crianza permisiva, partiendo por lo visto en el video.

Se obtuvo como respuesta de los padres; "esta forma de educar a los hijos no sirve...porque ellos se creen los papás, y no debe ser así...nosotros somos los padres y ellos nuestros hijos, deben respetarnos", "También es mala porque los hijos no nos harán caso cuando les demos alguna orden, siempre harán lo que quieran...pueden irse a las fiestas y hasta aprender cosas malas..." "mi hijo se volvería un delincuente si le dejo hacer lo que quiera... ya no quisiera tal vez ni estudiar...no, no, no, está crianza es muy perjudicial para ellos..."

Tema 4. Resultados de la crianza con amor y límites

Se proyectó un video que contó con escenas de padres, hablando entre ellos y otras personas hablando con sus hijos adolescentes, sobre lo que representa una crianza equilibrada, una crianza con amor y límites, como el nombre del programa.

La actividad que se realizó tuvo como fin, entre los grupos de padres, dar sus opiniones sobre la conducta generada por la crianza con amor y límites.

Se obtuvo como respuestas de los padres, después de la aplicación de las actividades;

"Yo antes era muy impulsivo, pero ahora respiro profundo y después me calmo o por lo menos trato de calmarme y escucho por qué actuaron así mis hijos", "cuando se portan mal mis hijos yo les castigo, pero a veces el castigo solo dura lo que dura mi enojo", "rara vez participamos en actividades porque mi esposa también trabaja y mis hijos ya 
no quieren salir con nosotros, ellos solo quieren estar con sus amigos", "si, yo le digo siempre que lo quiero y lo abrazo mucho...porque a los hijos hay que quererlos y demostrarles mucho amor".

\section{Sesión 2. Establecer reglas en la casa}

\section{Tema 1. ¿Cómo son los adolescentes?}

Se realizó la proyección de un video, donde las escenas mostraban cómo los adolescentes van cambiando tanto física como psicológicamente.

La actividad que se realizó tuvo como fin que cada uno de los padres diera su punto de vista sobre los cambios que observan en la adolescencia, tanto en el género femenino como masculino.

Se obtuvo como resultado, cambios a nivel físico y conductuales, entre ellos se mencionó: "son más altos", "más robustos", "le están saliendo pelitos en las axilas", "quieren estar solos" "pasa prendido al celular, y en cuanto a la familia "todo critican", "son contestones" entre otros.

\section{Tema 2. Reglas para nuestras familias}

Se proyectó un video acerca de la importancia de las reglas dentro de la familia. En la actividad que se desarrolló se les indicó a los padres presentes que mencionaran las conductas problemáticas de los hijos en el hogar y centro de estudio, en este sentido, se obtuvo como respuesta: "no ayudar en las labores domésticas" se convirtió en "ayudar en casa a lavar los platos después de comer", "no revisar tu mochila, para ver si te falta algo" se convirtió en "revisar tu mochila antes de irte a dormir, para que no te olvides ningún cuaderno", "mentir" se convirtió en "no mentir porque si no perderás mi confianza y ya no te creeré nada".

Tema 3. Usando afirmación en primera persona “me siento... cuando... porque... me gustaría.
Se desarrolló una actividad donde se leyó a los padres unas cuantas descripciones de situaciones presentadas en un cartel (que podían ver los padres) con el fin de que los padres expresaran sus sentimientos utilizando afirmaciones. En este sentido, los padres expresaron: "me siento enojado cuando no sacas la basura, porque es la regla que tenemos en casa, me gustaría que lo hagas por favor", "me siento triste cuando me contestas con insolencia, porque merezco que me respetes, me gustaría que no lo vuelvas a hacer", "me siento frustrada cuando no me ayudas con las tareas de la casa porque yo llego cansada de trabajar y solo te pido eso, me gustaría llegar a casa y que tu hayas ayudado con el quehacer"

Sesión 3. Protección para evitar las conductas de riesgo

Tema 1. Diferentes perspectivas en la relación a las conductas de riesgo

En esta actividad, se explicó al grupo que se va trazar una línea imaginaria en el piso con dos extremos, un extremo representa a los padres que creen en la abstinencia total de la conducta (consumo de alcohol, tabaco, drogas, actividad sexual responsable con preservativos) y en el otro extremo corresponde a los padres que piensan que no debe haber ningún control sobre esa misma conducta de sus hijos. Por lo que, cada uno eligió su lugar, y explicaron el motivo por el cual, eligieron esa opción. De esta forma, se obtuvo como respuestas, "porque en mi casa nadie bebe alcohol y no estoy de acuerdo que mi hijo lo haga", "considero que primero como padre debo explicarle lo malo de consumir cualquier tipo de droga, como le podría afectar y arruinar todos sus sueños".

Posterior, se interrogó a los padres, sobre la edad adecuada en que los adolescentes son 
suficientemente maduros para tener las conductas mencionadas anteriormente. Tomando en cuenta si tiene hija o hijos. En este sentido, se obtuvo como respuesta: "una vez que cumplan la mayoría de edad el podrá decidir lo que es mejor para él... pero considero importante que antes de eso debo explicarle lo malo que le podría acarrear", "por mí ojalá nunca pruebe el alcohol ni ninguna otra droga. Pero sé que pronto crecerá y tal vez vaya a alguna fiesta y ahí le pueden ofrecer, por eso es importante que ya lo vaya preparando para que él sepa decir que NO", "no, no es lo mismo para las niñas porque ellas son más frágiles y como padre de una niña creo que la mujer no debe tomar porque se ve feo...además los hombres se podrían aprovechar de ella".

Tema 2. Peligros con el consumo de tabaco y drogas, el abuso del alcohol y la desinformación acerca de las conductas sexuales de riesgo

En esta actividad, se les pidió a los padres que enumeraran las cosas que podrían ser graves problemas para sus hijos si beben, si usan drogas, o si no tienen información sobre las conductas sexuales de riesgo. Por lo que se obtuvo como respuesta: "abandono de los estudios y menores oportunidades de alcanzar sus metas y sueños", "sexo no deseado, embarazo en la adolescencia y contagio de alguna enfermedad de transmisión sexual o VIH/SIDA", "pueden caer en la delincuencia y actividades antisociales como participación en pandillas, juntarse con malas influencias, etc."

\section{Sesión 4. Conexiones con la comunidad}

Tema 1. ¿Por qué es importante la escuela?

Se proyectó un video sobre la importancia de la escuela con la finalidad de que los padres posteriormente dieran su opinión al respecto de la importancia de la escuela.
De esta forma, se obtuvo como respuestas; "seguir en la escuela puede ayudarles a conseguir un mejor trabajo", "pueden ganar más dinero", "los ayudará a mantenerse lejos de problemas", "los ayudará a cumplir sus sueños". Después, se les volvió a realizar otra interrogante a los padres, de cómo pueden ayudar a sus hijos a mantenerse interesados por la escuela; por lo que se obtuvo como respuesta; "visitar la escuela y al profesor para preguntarle sobre el desenvolvimiento de mi hijo en la escuela", "motivar a los adolescentes a que sigan estudiando", "interesarme más por sus cosas preguntarle en qué lo puedo ayudar".

Tema 2. Hacer que nuestra comunidad sea segura para nuestros hijos

En esta actividad se obtuvo una lista de problemas o conflictos que los padres consideraron que existían en su comunidad, y afectaban a los adolescentes, de esta manera, se señalo como principales el alcoholismo, drogas, delincuencia, entre otras.

Tema 3. ¿Dónde conseguir ayuda?

Después de la proyección del video sobre los lugares donde se pueden conseguir ayuda, se les indicó a los padres responder unas series de preguntas, relacionadas al video. Por lo que se obtuvo como respuestas; "sí debo ir a la alcaldía, FELCC, FELCV, INTRAID”, "por vergüenza...porque a veces no queremos que la gente se entere de mis problemas", "porque no hay confianza en las autoridades".

Así que, se reforzó indicando otras instituciones para solicitar ayuda como ser: Defensoría, SLIM, Defensor del pueblo, Fiscalía, FELCC, FELCV, entre otros. 


\section{CONCLUSIONES}

De acuerdo a los resultados obtenidos podemos concluir indicando que se cumplió el objetivo trazado para esta actividad. Por lo tanto, se puede decir que, los estudios abren oportunidades de progreso a los jóvenes, los padres deben ayudar a sus hijos a mantener el interés en la escuela, con las construcciones de redes de apoyo se puede crear mejores oportunidades locales para los adolescentes en el cuidado de la salud, opciones de recreación, apoyo escolar, entre otros aspectos. A su vez, una familia fuerte es la que tiene el coraje de pedir ayuda y unirse a otros para salir adelante.

Además, los padres, a partir de estas actividades reconocieron que las relaciones con sus hijos mejoraron; también muchos de ellos comentaron que les había gustado las actividades ya que pueden explicarles a sus hijos lo negativo del consumo de alcohol o drogas.

Para finalizar, con el programa familia fuerte, los padres, el lema "con amor y límites seremos una mejor familia", ayuda a los padres a enfatizar lo aprendido.

\section{REFERENCIAS}

Bandura, A. (1983). Principios de modificación de conducta. Salamanca- España, Ediciones Sígueme

Barber, B., y Olsen, J. (1997). Socializations in context: Connection, regulations, and autonomy in the family, school, and neighborhood, and with peers. Journal of Adolescent Research, 12, 287-315

Bartholomew, K. (2005). Mapeo de Intervención: Diseño de Teoría y Evidencia
Brazalton, T.(2006). Extraído de:www.parentsaction. org/wpcontent/uploads/2017/04/PAFC_Discbklt-Span.pdf

Brienbauer. C., y Maddaleno, M. (2008).Jóvenes: Opciones y cambios. Promoción de conductas saludables en los adolescentes. Publicación Científica y Técnica N ${ }^{\circ}$ 594. WDC. España

Bronfenbrenner, U. (1982). El modelo ecológico y el desarrollo humano. España

Cangas, A., y Moldes, P. (2009). Claves: habla con tus hijos. México: koala

González, M.; Hidalgo, M., y Moreno, C. (1998). La vida en Familia. Cuadernos de pedagogía, 274, 50-55

INTRAID. Extraído de http://www.elpaisonline. com/index.php/sociales- 2/item/33437-tarijaocupa-el-primer-lugar-nacional-en-consumode-alcohol

Kumpfer, L. (2006). Fortalecimiento de las familias de los Estados Unidos: Estrategias ejemplares para padres y familias para la prevención de la delincuencia. Oficina de Justicia Juvenil y Prevención de la Delincuencia. WDC. E.E.U.U.

Malkus. (1994). Extraído de https://books.google. com/books? Isbn=8415538731

Muss, R. (1996).Teorías sobre el adolescente. Edición N6. Mcgraw-Hill. Nueva York

OPS/OMS (2013). La familia y la salud. $132^{\circ}$ Sesión del Comité Ejecutivo. WDC

Pichon, E (2009). Teoría del vínculo, Colección psicología contemporánea. Proyectos de prevención de la familia basados en las asociaciones entre la comunidad y las universidades: Hacia un aumento de las intervenciones preventivas. Visión, Buenos Aires

UNODOC. (2016). Informe Mundial sobre las Drogas, Oficina de las Naciones Unidas contra la Droga y el Delito 